DOI:10.2478/rrlm-2020-0009

\title{
A rare case of acute myeloid leukemia with ARHGEF12 (LARG, 11q23.3) and MAPRE1 (EB1, 20q11.21) fusion gene in an elderly patient
}

\author{
Ioan Macarie ${ }^{1}$, Florin Tripon ${ }^{1 *}$, Bogdana Dorcioman², Melania Macarie ${ }^{1}$ \\ 1. George Emil Palade University of Medicine, Pharmacy, Science, and Technology of Targu Mures, \\ Romania \\ 2. Targu Mures Clinical County Emergency Hospital, Central Laboratory, Romania
}

\begin{abstract}
Introduction. We report one elderly patient diagnosed with a rare subtype of acute myeloid leukemia (AML) and also with a very rare fusion gene involving ARHGEF12 (LARG, 11q23.3) and MAPRE1 (EB1, 20q11.21) genes. Material and methods. Clinical examination and routine analysis were performed including peripheral blood smear, immunophenotyping of the peripheral blood by flow cytometry and several molecular analyses. Results. Peripheral blood smear showed $80 \%$ blasts with round and some with convoluted nuclei, with basophilic cytoplasm, identified as monoblast and the majority of cells as promonocytes. Peripheral blood immunophenotyping was consistent with monocytic differentiation. Molecular analysis was negative for FLT3 ITD, FLT3 D835, NPM1, and DNMT3A R882 mutations. Multiplex ligation-dependent probe amplification revealed no copy number aberration. Ligation-dependent reverse transcription polymerase chain reaction (LD-RT-PCR) analysis identified the presence of one gene fusion between ARHGEF12 (LARG, 11q23.3) and MAPRE1 (EB1, 20q11.21) genes. The patient had no significant comorbidities, the renal function was normal and Eastern Cooperative Oncology Group performance status was 2 at diagnosis and 1 after treatment. She was treated with decitabine. She became transfusion independent and a reduction of the number of blasts was obtained. Conclusions. The outcome of our AML patient was favorable but other patients with fusion genes involving ARHGEF12 (LARG, 11q23.3) and MAPRE1 (EB1, 20q11.21) should be reported, contributing to a better characterization of the disease, to monitor the minimal residual disease and in the end to more targeted treatment options. LD-RT-PCR represent a valuable multiplex technique for fusion gene analysis.
\end{abstract}

Keywords: AML, fusion gene, ARHGEF12, MAPRE1

Received: 25 th December 2019; Accepted: 11 th January 2020; Published: $15^{\text {th }}$ January 2020

*Corresponding author: Florin Tripon, George Emil Palade University of Medicine, Pharmacy, Science, and Technology of Targu Mures, Romania. E-mail: tripon.florin.2010@gmail.com 


\section{Introduction}

Acute myeloid leukemia (AML) is the second most common leukemia and the most common acute leukemia in adults, with progressively increasing incidence with advancing age. The median age at diagnosis is 67 years (1). Only 10\% of older adults are long-term survivors (2). It is a clonal disease originating in hematopoietic stem cells, usually with multilineage potential. The leukemic blasts proliferate in the bone marrow and peripheral blood. AML is a heterogeneous neoplasm with a large number of recognized genetic abnormalities. Most common are identified as balanced translocations, detected by karyotyping, fluorescence in-situ hybridization (FISH), polymerase chain reaction (PCR) or other molecular methods. World Health Organization (WHO) classification includes AML with recurrent cytogenetic abnormalities as a separate category(3). Also, fusion genes are very frequently found in hematological malignancies, including AML patients. Commonly, fusion genes are generated by chromosomal rearrangements but also they can result as a consequence of abnormal transcription (4). Fusion between myeloid/lymphoid or mixed lineage leukemia gene [MLL (KMT2A), 11q23.3] and Microtubule-associated protein, RP/EB family, member 1 gene [MAPRE1 (EB1), 20q11.21] genes was reported until now only in one patient with acute lymphoblastic leukemia (ALL) (5) and between MLL1 (KTMT2A) and/or Rho guanine nucleotide exchange factor 12 gene [ARHGEF12 (LARG), 11q23.3] genes in one AML patient (6).

In this case study, we report an AML patient with a fusion gene involving ARHGEF12 (LARG, 11q23.3) and MAPRE1 (EB1, 20q11.21) genes. The patient signed the informed consent for medical procedures and for reporting the case. A separate genetic testing informed consent was also signed by the patient. The Ethics Committee of the Târgu Mureș Clinical County Emergency
Hospital, Romania, reviewed and approved the study (decision no. 36963/2019).

\section{Case report}

We report a case of an 88-year-old woman, diagnosed with de novo AML. She was anemic, with increased white blood cell count (WBC), with $80 \%$ blasts with monoblast lineage morphology and low platelet number. Her WBC was $26.33 \times 10^{3} / \mu \mathrm{L}$ (normal value $3.6-10 \times 10^{3} / \mu \mathrm{L}$ ), hemoglobin $7.00 \mathrm{~g} / \mathrm{dL}$ (normal value $12-15 \mathrm{~g}$ / $\mathrm{dL}$ ), platelets 49.00 (normal value $150-450 \times 10^{3}$ / $\mu \mathrm{L})$. Peripheral blood smear showed $80 \%$ blast equivalents with round and some with convoluted nuclei, with basophilic cytoplasm, identified as monoblast and the majority of cells as promonocytes (Figure 1).

Immunophenotyping of the peripheral blood by flow cytometry was performed. The blood sample was stained using monoclonal antibodies, then the erythrocytes were lysed and then washed. The remaining WBC were analyzed by direct immunofluorescence technique for surface markers. The samples were analyzed by multiparameter flow cytometry on Becton Dickinson FACS CALIBUR device (Becton Dickinson, USA). Peripheral blood immunophenotyping was consistent with monocytic differentiation. The population of blasts was positive for CD13, CD33, CD15, HLA-DR, CD11b, CD14, CD64, and CD36. The expression of CD56 was weak and the blasts were negative for CD34, CD117, CD3, CD5, CD7, CD19, CD10, and CD22 (Figure 2).

Genetic analysis was done from peripheral blood. Genomic DNA (gDNA) was isolated from leukocytes (PureLink Genomic DNA kit, ThermoFisher Scientific, USA) and used for somatic mutation analysis and for copy number aberration (CNA) investigation by Multiplex ligation-dependent probe amplification (MLPA) analysis. 

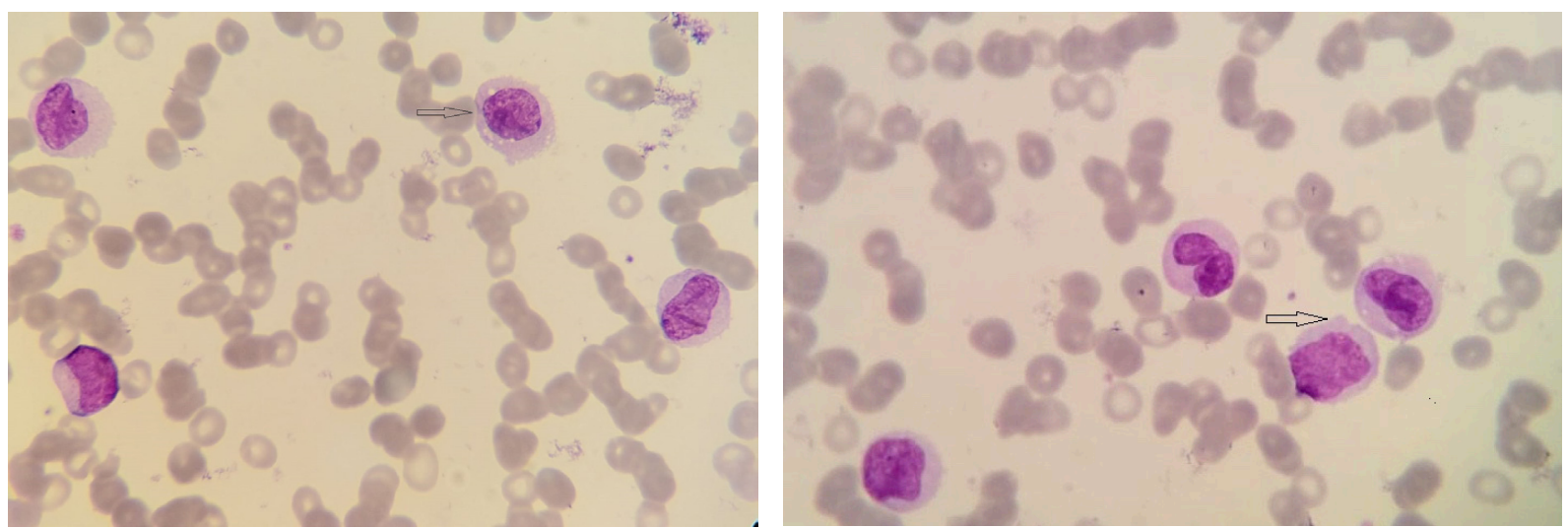

Fig. 1. Morphology of the blasts stained with May-Grunwald Giemsa (100x); A-monoblast morphology, rounded nuclei, basophilic cytoplasm; B-promocyte morphology, more irregular nuclei, less basophilic cytoplasm, monocytic lineage morphology.

FMS-like tyrosine kinase-3 (FLT3) internal tandem duplication (FLT3 ITD) and FLT3 D835, Nucleophosmin 1 (NPM1, mutations type A-D) and DNA (cytosine-5)-methyltransferase 3A (DNMT3A, R882 codon) mutations were analyzed according to our protocol published before (7). Fragment analysis and GeneMapper software (ThermoFisher Scientific, USA) were used in order to calculate the Variant-Allele Frequency/Allele Ratio for FLT3 ITD and NPMI mutations.

MLPA analysis was used in order to detect CNAs and aneuploidy identification as previously published $(8,9)$ being well known that MLPA represents a useful analysis for AML patients $(8$, 10). For aneuploidy detection, in addition to the probemix (SALSA P-036 Subtelomere mix 1) used in a published protocol (8), we also used SALSA MLPA P070 Subtelomeres Mix 2B, P181 Centromere mix 1 and P182 Centromere mix 2 (MRC Holland, Amsterdam). Salsa MLPA P377 Hematological malignancies mix and P335 ALL-IKZF1 probemixes were used to analyze chromosomal regions that are currently known to have significant importance on AML risk stratification, diagnostic or prognostic. Ligation-dependent reverse transcription polymerase chain reaction (LD-RT-PCR) analysis technique was used in order to detect specific gene fusion (more than 50) for acute leukemias as previously reported (11). We adapted the protocol used by Ruminy P et al (11) and we performed the sequencing reaction by capillary electrophoresis. Also, we removed the primers for NPM1 (type A-D mutations) from this protocol. The total RNA was isolated from peripheral blood (QuickRNA Whole Blood kit, ZymoResearch) and the reverse transcription was performed according to the manufacturer recommendations (High Capacity cDNA Reverse Transcription Kit, ThermoFisher Scientific, USA). Five microliters of complementary DNA (cDNA) were used for LD-RT-PCR analysis.

Molecular analysis was negative for FLT3 ITD, FLT3 D835, NPM1, and DNMT3A R882 mutations. MLPA results were normal, without CNAs. LD-RT-PCR analysis revealed the presence of one gene fusion between ARHGEF12 (LARG, 11q23.3) and MAPRE1 (EB1, 20q11.21) genes (Figure 3). Unfortunately, we were unable to perform a standard karyotyping or FISH analysis for confirmation. Bone marrow aspiration was not performed due to patient age and due to the fact that the percentage of blasts in the 

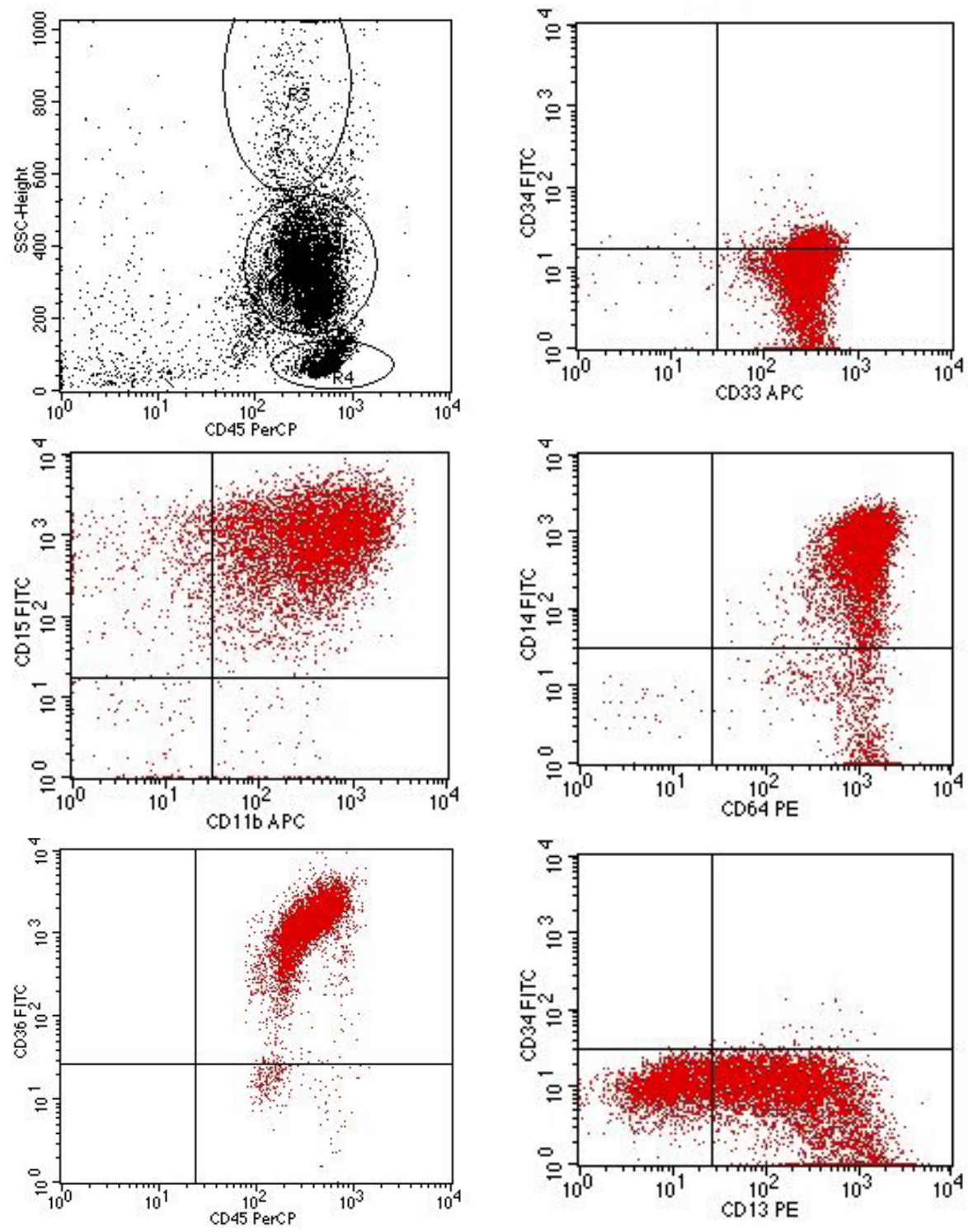

Fig. 2. Immunophenotype of blasts (red) detected by flow cytometry in the peripheral blood sample (surface markers) with expression of monocyte lineage differentiation antigens.

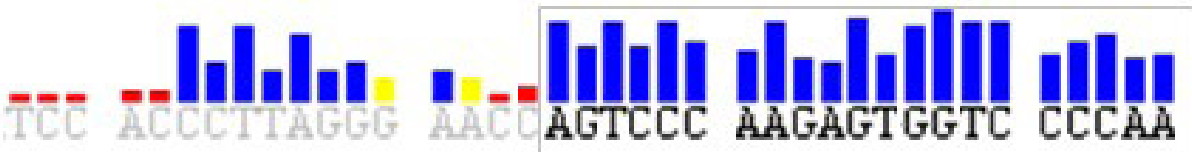

Fig. 3. Sequencing results of LD-RT-PCR product. The DNA sequence specific for $M A P R E 1$ (EB1, 20q11.21) gene is wrriten with grey color followed by the DNA sequence specific for $A R H G E F 12$ (LARG, 11q23.3) gene, written in black and framed in the border. 
peripheral blood was very high and it allowed a morphologic and immunophenotype-based diagnosis.

In our patient, AML diagnosis was not preceded by myelodysplastic syndrome (MDS). The final diagnosis was de novo acute monocytic leukemia with ARHGEF12 (LARG, 11q23.3) and MAPRE1 (EB1, 20q11.21) gene fusion.

In old patients with AML, standard treatment with remission induction therapy is generally not indicated and in patients older than 80 years, regardless of Eastern Cooperative Oncology Group (ECOG) performance status, standard chemotherapy is not beneficial for survival. Our patient had no significant comorbidities, the renal function was normal and ECOG performance status was 2 . She was treated with decitabine (Dacogen) $20 \mathrm{mg} / \mathrm{m}^{2} /$ day for 5 days in a 1 -hour infusion, with cycles repeated every 28 days. She became transfusion independent and a reduction of the number of blasts in the peripheral blood was obtained. She continues to tolerate the treatment well and has an ECOG of 1. Hypomethylating agents (HMA), especially decitabine, are active in AML with unfavorable genetic risk factors.

\section{Discussions}

Acute monocytic leukemia is a rare subtype of AML, being responsible for less than $5 \%$ of AML cases. Moreover (compared with our AML case), it is more common in young patients being one of the most frequent subtypes of AML in children younger than 2 years of age (12). As reported in the literature, in acute monocytic leukemia, the predominating cells are promonocytes that have a more irregular and convoluted nuclei and a less basophilic cytoplasm (3). Gingival hypertrophy and other manifestation of extramedullary disease are often seen in monocytic acute leukemia. In this case study, we reported one elderly patient diagnosed with a rare subtype of AML and also with a very rare fusion gene. ARHGEF12 (LARG, 11q23.3) and MAPRE1 (EB1, 20q11.21) gene fusion according to the best of our knowledge was not reported previously in the literature on AML patients. The fusion between MLL (KMT2A) and MAPRE1 (EB1) genes was reported until now only on one patient with ALL (5). MLL (KMT2A) gene is situated near to our implicated gene, ARHGEF 12 $(L A R G)$. Both genes are localized on 11q23.3 chromosomal region. As the ALL patient with the mentioned fusion gene reported by $\mathrm{Fu}$ JF et al (5), our patient presented high WBC count and blasts percentage and some differences in immunophenotypic analysis. Unfortunately, Fu JF et al (5) were not able to analyze the presence of CNAs and gene mutations and to describe the patient outcome because their patient left the hospital (5). As a consequence, more reports are needed in order to evaluate the clinical impact of ARHGEF12 (LARG, 11q23.3) and MAPRE1 (EB1, 20q11.21) gene fusion on AML patients, and not only. Fu JF et al (5) used genes analysis molecular target techniques for fusion, only for the mentioned genes. In this case study, we used an adapted multiplex protocol but primers for MLL (KMT2A, 11q23.3) were not included. One of our limitations is the fact that we were unable to clarify the cause of the fusion and also the breakpoints. It is possible for a larger chromosomal region to be implicated, including $M L L$ (KMT2A) gene reported by Fu JF et al (5). As reported in the literature $(3,4)$ and also on the similar ALL case reported by Fu JF et al (5), commonly fusion genes are the result of translocations, which frequently are a result of deficient DNA damage-repair pathway, which may also be a result of other primary factors such as reactive oxygen species $(3-5,13)$.

AML with 11q23 abnormalities was reported to be associated with monocytic differentiation (14). Anomalies of chromosome 11q23.3 may also be present in AML with myelodysplasia-related changes and in therapy-related AML (3). More than 120 different translocations ( $\mathrm{t}$ ) involving KMT2A were previously described in 
pediatric and adult leukemia, both lymphoblastic and myeloid (14).

A separate category in WHO classification is mixed-phenotype acute leukemia with (v;11q23.3). It should be differentiated from ALL cases with $11 \mathrm{q} 23.3$ - KMT2A gene mutation in lymphoid blasts with co-expressions of myeloid-associated antigens. The WBC count is increased, the blast population is dimorphic at microscopy and immunophenotype is positive for CD19 and CD15 and negative for CD10. Usually, myeloid features characteristic for monoblastic phenotype are seen in a separate population. Cases with 11q23.3 deletions should not be considered in this category. KMT2A translocation may be the only abnormality or may co-exist with other genetic mutations (15).

Elderly patients with AML have a poor response to standard induction chemotherapy. Blood transfusion for anemia, platelet transfusions for hemorrhages due to thrombocytopenia, antibiotics for infections, hydroxyurea for hyperleukocytosis remain the best available conservative treatment in severely ill patients. Treatment with low-dose cytarabine (LDAC) is not efficient in patients with genetic abnormalities. HMA, decitabine and azacytidine, have usually low response rates and 3-4 months are needed to achieve a response. Currently the optimal treatment for elderly patients with AML ( $>65$ years and ineligible for intensive chemotherapy or $>75$ years) is association of oral bcl-2 inhibitor venetoclax with a HMA such as decitabine or azacytidine (16).

Recurrent cytogenetic abnormalities involving $K M T 2 A$ gene on chromosome 11q23 were reported in $3-4 \%$ of adult AML. The prognostic significance of the rare translocations, other than $\mathrm{t}(9 ; 11)$ were rarely reported. The $\mathrm{t}(11 ; \mathrm{v})(\mathrm{q} 23 ; \mathrm{v})$ is an independent poor risk factor and the eligible patients will benefit from allogeneic stem cell transplantation in first remission (17).

KMT2A gene mutations have different prognostic value depending on the fusion partner and testing for these anomalies is incorporated in some protocols (11).

In our case, the patient outcome was favorable, with at least partial response. Involvement of the 11q23 (KMT2A gene) with other partner genes than MLLT3 on chromosome 9p21 is associated with a poor prognosis. No series of AML patients with ARHGEF12 fusion was reported yet and a response to treatment, based on this characteristic is difficult to interpret. In elderly patients, as our patient, the treatment options are limited to LDAC and HMA associated or not with venetoclax. Lack of response to any of these treatments was as yet not reported. In younger, transplant eligible patients, poor prognostic genetic risk is an indication for allotransplant in first complete remission. More reports are needed in order to clarify the role of ARHGEF12 (LARG, 11q23.3) and MAPRE1 (EB1, 20q11.21) fusion gene in AML patients.

As reported by Shih et al (18), the 11q23.3 translocation is not evident on standard karyotype in some cases. Sometimes FISH or other molecular techniques are needed to identify the variant translocations (18). Thus, we suggest LD-RT-PCR analysis as the first approach for fusion gene investigation. Moreover, we consider LD-RT-PCR analysis as a rapid and low-cost technique that may be performed for the first investigation of leukemia patients. Our AML patient did not present CNAs, but as previously reported, we agree that MLPA represents a useful analysis for the detection of CNAs with clinical significance (8-10, 19-20).

\section{Conclusions}

Full evaluation of all AML patients should be made in clinical practice, including molecular analysis. After examination of the peripheral blood and bone marrow aspirate by microscopy and immunophenotyping for diagnosis, the molecular analysis adds more information about 
disease risk and leukemia pathogenesis. The outcome of our AML patient was favorable but other patients with fusion genes involving $A R$ HGEF12 (LARG, 11q23.3) and MAPRE1 (EB1, 20q11.21) should be reported, contributing to a better characterization of the disease, to monitoring the minimal residual disease and in the end to more targeted treatment options. LD-RTPCR represents a valuable multiplex technique for fusion gene analysis.

\section{Abbreviations}

AML-acute myeloid leukemia

FISH-fluorescence in-situ hybridization

PCR-Polymerase chain reaction

WHO-World Health Organization

MLL-myeloid lymphoid leukemia/mixed lineage leukemia

ALL-acute lymphoblastic leukemia

WBC-White blood cells

gDNA-genomic DNA

CNAs-copy number aberrations

MLPA-Multiplex ligation-dependent probe amplification

FLT3-FMS-like tyrosine kinase 3

ITD-internal tandem duplication

NPM1-nucleophosmin 1

DNMT3A-DNA methyltransferase 3A

LD-RT-PCR-ligation-dependent reverse-transcription polymerase chain reaction

cDNA-complementary DNA

MDS-myelodysplastic syndrome

ECOG-Eastern Cooperative Oncology Group

HMA-hypomethylant agents

LDAC-low dose arabinoside cytarabine

\section{Acknowledgments}

Part of the genetic analyses were supported by an internal grant of the George Emil Palade University of Medicine, Pharmacy, Science and Technology of Targu Mures, Romania, no. 15609/13/29.12.2017.

\section{Authors' contributions}

MI - conceptualization, data collection, interpretation of data, writing - original draft, review; FT - performing genetic analysis, design, interpretation of data, writing - review \& editing; $\mathrm{BD}$ - immunophenotyping, interpretation of data, writing;

MM - resources, supervision, original draft preparation.

All authors were involved in drafting and reviewing the submitted manuscript for important intellectual content.

\section{Conflict-of-Interest}

The authors declare that there is no conflict of interest.

\section{References}

1. Almeida AM, Ramos F. Acute myeloid leukemia in the older adults. Leuk Res Rep. 2016;6:1-7. DOI: 10.1016/j.lrr.2016.06.001

2. Ofran Y, Tallman MS, Rowe J. How I treat acute myeloid leukemia presenting with preexisting comorbidities. Blood. 2016;128(4):488-96. DOI: 10.1182/ blood-2016-01-635060

3. Arber DA, Orazi A, Hasserjian R, Thiele J, Borowitz MJ, Le Beau MM et al. The 2016 revision to the World Health Organization classification of myeloid neoplasms and acute leukemia. Blood. 2016;127(20):2391405. DOI: 10.1182/blood-2016-03-643544

4. Wang Y, Wu N, Liu D, Jin Y. Recurrent Fusion Genes in Leukemia: An Attractive Target for Diagnosis and Treatment. Curr Genomics. 2017;18(5):378-384. DOI: 10.2174/1389202918666170329110349

5. Fu JF, Hsu HC, Shih LY. MLL is fused to EB1 (MAPRE1), which encodes a microtubule-associated protein, in a patient with acute lymphoblastic leukemia. Genes Chromosomes Cancer. 2005;43(2):206-10. DOI: 10.1002/gcc. 20174

6. Kourlas PJ, Strout MP, Becknell B, Veronese ML, Croce CM, Theil KS et al. Identification of a gene at 11q23 encoding a guanine nucleotide exchange factor: evidence for its fusion with MLL in acute myeloid leu- 
kemia. Proc Natl Acad Sci U S A. 2000;97(5):2145-50. DOI: $10.1073 /$ pnas.040569197

7. Tripon F, Crauciuc GA, Moldovan VG, Bogliș A, Benedek IJ, Lázár E et al. Simultaneous FLT3, NPM1 and DNMT3A mutations in adult patients with acute myeloid leukemia - case study. Rev Romana Med Lab. 2019;27(3):245-54. DOI: 10.2478/rrlm-2019-0022

8. Vázquez-Reyes $\mathrm{A}$, Bobadilla-Morales $\mathrm{L}, \mathrm{Bar}-$ ba-Barba C, Macías-Salcedo G, Serafín-Saucedo G, Velázquez-Rivera ME et al. Aneuploidy identification in pre-B acute lymphoblastic leukemia patients at diagnosis by Multiplex Ligation-dependent Probe Amplification (MLPA). Leuk Res. 2017;59:117-123. DOI: 10.1016/j.leukres.2017.05.022

9. Bănescu C. Do we really need genetic tests in current practice? Rev Romana Med Lab. 2019;27(1):9-14. DOI: $10.2478 / \mathrm{rrlm}-2019-0010$

10. Bănescu C, Tripon F, Trifa AP, Crauciuc AG, Bogliș A, Lazar E et al. Presence of copy number aberration and clinical prognostic factors in patients with acute myeloid leukemia: an analysis of effect modification. Pol Arch Intern Med. 2019;129(12):898-906. DOI: 10.20452/pamw.15093

11. Ruminy P, Marchand V, Buchbinder N, Larson T, Joly $\mathrm{B}$, Penther B et al. Multiplexed targeted sequencing of recurrent fusion genes in acute leukaemia. Leukemia. 2016;30(3):757-60. DOI: 10.1038/leu.2015.177

12. Verschuur A. Acute monocytic leukemia. Orphanet Encyclopedia 2004. Available at https://www.orpha.net/ data/patho/GB/uk-AMLM5.pdf. (Last time accesed on 22 dec. 19)

13. Bănescu C, Iancu M, Trifa AP, Cândea M, Benedek Lazar E, Moldovan VG et al. From Six Gene Polymorphisms of the Antioxidant System, Only GPX Pro198Leu and GSTP1 Ile105Val Modulate the Risk of Acute Myeloid Leukemia. Oxid Med Cell Longev. 2016;2016:2536705. DOI: 10.1155/2016/2536705

14. Arber DA, Brunning RD, Le Beau MM, Falini B,
Vardiman JW, Porwit A et al. Acute myeloid leukemia with recurrent genetic abnormalities. In Swerdlow SH, Campo E, Harris NL, Jaffe ES, Pileri SA, Stein H, et al. WHO classification of tumors of haematopoietic and lymphoid tissues, Lyon: IARC. 2017, p 136-7.

15. Borowitz MJ, Bene MC, Harris NL, Porwit A, Matutes E. Arber DA. Acute leukemias of ambiguous lineage. In Swerdlow SH, Campo E, Harris NL, Jaffe ES, Pileri $\mathrm{SA}$, Stein H, et al. WHO classification of tumors of haematopoietic and lymphoid tissues, Lyon: IARC. 2017, p 183-4.

16. DiNardo DC, Pratz K, Pullarkat V, Jonas BA, Arellano $\mathrm{M}$, Becker PS et al. Venetoclax combined with decitabine or azacytidine in treatment naïve, elderly patients with acute myeloid leukemia. Blood. 2019;133(1):717. DOI: 10.1182/blood-2018-08-868752

17. Chen Y, Kantarjian H, Pierce S, Faderl S, O'Brien S, Qiao W et al. Prognostic significance of 11q23 aberrations in adult acute myeloid leukemia and the role of allogeneis stem cell transplantation. Leukemia. 2013;27:836-42. DOI: 10.1038/leu.2012.319

18. Shih LY, Liang DC, Fu JF, Wu JH, Wang PN, Lin TL et al. Characterization of fusion partner genes in 114 patients with de novo acute myeloid leukemia and MLL rearrangement. Leukemia. 2006 Feb;20(2):218-23. DOI: $10.1038 /$ sj.leu.2404024

19. Bogliş A, Tripon F, Bănescu C. The utility of molecular genetic techniques in craniosynostosis cases associated with intellectual disability. Rev Romana Med Lab. 2018;26(4):471-7. DOI: 10.2478/rrlm-2018-0033

20. Crauciuc GA, Tripon F, Bogliş A, Făgărăşan A, Bănescu C. Multiplex ligation dependent probe amplification - A useful, fast and cost-effective method for identification of small supernumerary marker chromosome in children with developmental delay and congenital heart defect. Rev Romana Med Lab. 2018;26(4):461-70. DOI: $10.2478 / \mathrm{rrlm}-2018-0032$ 